\title{
The Control and Simulation of ADRC-based for VSC-HVDC System
}

\author{
Jianyuan Dong, Yanan Tian, Xue Zhang
}

School of Mechanical and Electrical Engineering, Xi'an University of Architecture \&Technology, Xi'an 710055, China

Keywords: VSC-HVDC; ADRC control; SPWM; PID control; NLSEF.

\begin{abstract}
The high voltage direct current transmission based on voltage source (VSC - HVDC) technology has been widely used in transmission areas. Aimed at the problem of the low Steadystate accuracy and weak anti-interference ability for the traditional PI control, a control strategy based on ADRC was proposed. The mathematical model of the system was set up. Sending system and reception system were designed by ADRC. Fall function was used for out ring of sending system and PI control was used for inner ring. DC voltage control was used for out ring of reception system, Current and reactive power control was used for inner ring. With Matlab/Simulink the designed controller were simulated and the results were better than the traditional controller in control performance.
\end{abstract}

\section{Introduction}

With the energy shortage and environmental pollution issues have become increasingly severe, wind, solar and other renewable energy continues to expand the scale, its inherent dispersion, small nature, away from the load centre and other features, making traditional HVDC technology seems very economical networking. Some offshore drilling platforms, isolated islands and other passive load, mostly using expensive local power plant, not economic, but also pollute the environment [1]. In addition, the rapid increase in urban electricity load, the need to continue to expand the capacity of the grid. This requires a new HVDC technology to solve these problems. The use of inverter and pulse width modulation (PWM) voltage source based on the type of device can be turned off new technology HVDC technology (VSC-HVDC) can solve this problem [2]. From the operation of the technical characteristics and practical engineering point of view, it is suitable for renewable energy grid, power islands, urban power grid and other fields. Using VSC-HVDC power supply to a number of islands, may limit the short circuit capacity of the system, while the DC line in terms of investment, operating costs, long-distance transmission equipment, etc. do not need to add compensation with respect to the AC line has advantages. For some large urban power supply system, the adoption of VSC-HVDC, can not only improve the quality of the supply of electricity, but also can improve system stability [3]. Therefore, the VSC-HVDC will play a huge role in the next city Uprating baking.

\section{Establish the mathematical model of VSC-HVDC system}

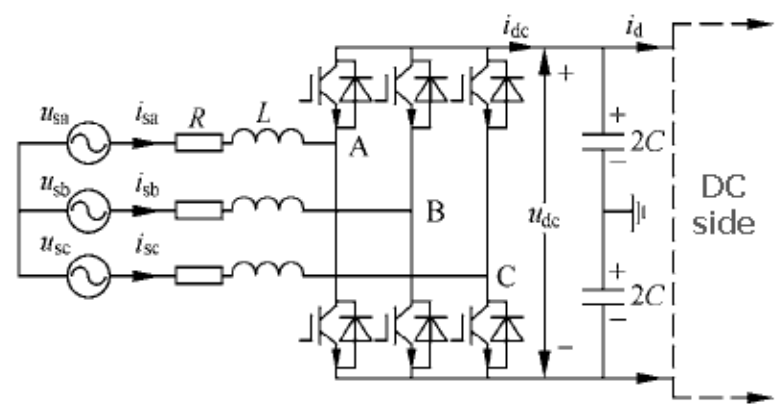

Fig.1 The structure of voltage source converter

Set up a three-phase grid voltage balance, according to Figure 1's Topology, can obtain synchronously rotating coordinate system's Mathematical model. 


$$
\begin{aligned}
& L \frac{d i_{s d}}{d t}-w L \dot{s}_{s_{q}}+R i_{s d}=u_{s d}-u_{d} \\
& L \frac{d i_{s q}}{d t}-w L \dot{i}_{b d}+R i_{s d}=u_{s q}-u_{q} \\
& C \frac{d u_{d c}}{d t}=\frac{3}{2}\left(s_{d} i_{s d}+s_{q} i_{s q}\right)-i_{d} \ldots
\end{aligned}
$$

In this formula, $\mathrm{u}_{\mathrm{sd}}, \mathrm{u}_{\mathrm{sq}}$ represent $\mathrm{d}$, q-axis component of the grid voltage, $\mathrm{u}_{\mathrm{d}}, \mathrm{u}_{\mathrm{q}}$ represent $\mathrm{d}$, q-axis component of VSC AC side voltage fundamental. $i_{s d}, i_{s q}$ represent $d$, q-axis component of grid current. In synchronous rotating coordinate system [4], active and reactive power can be expressed as

$$
\left\{\begin{array}{l}
p_{s}=\frac{3}{2}\left(u_{s d} i_{d}+u_{s q} i_{q}\right) \\
q_{s}=\frac{3}{2}\left(u_{s d} i_{q}-u_{s q} i_{d}\right)
\end{array}\right.
$$

When the $\mathrm{d}$-axis positioning to the grid voltage vector, that $\mathrm{u}_{\mathrm{sq}}=0$.The formula (4) can be written as

$$
\left\{\begin{array}{l}
p_{s}=\frac{3}{2} u_{s} i_{d} \\
q_{s}=\frac{3}{2} u_{s d} i_{q}
\end{array}\right.
$$

By the formula (3) shows, control the $i_{d}, i_{q}$ can adjust the active and reactive power of converter stations and communication systems [5].

\section{Design of VSC-HVDC system based on ADRC controller}

PI control of VSC-HVDC system is a method that it adjust controller's parameters, so that the system reaches a steady state. But the system have the problem of the low Steady-state accuracy and weak anti-interference ability. It needs depending on the operating conditions to continue to adjust the control parameters [6]. Aimed at these problems, this paper presents a technique of ADRC. Its controller mainly consist of TD, ESO and NLSEF. First, TD can quickly track the system's input signal without overshoot and it can give a good differential signal. Secondly, ADRC system's total disturbance consist of the system model uncertainty and system interference. It directly detect the internal disturbance and the external disturbance, rather than specific to distinguish them. ESO were estimated by the system state and disturbance. Finally, disturbance component's compensation are obtained by NLSEFL [7].As ADRC can be applied to the decoupling control of multivariable systems, it don't require the amount of computation, and have a good robustness. Therefore, it is applied to the VSC-HVDC converter station controller's outer control, the inner part still use PI [8].

\section{Design of sending system controller}

Sending system use active control strategy. The inner current controller complete decoupling control by PI controller and outer controller design includes the active power and reactive power control controller[8].During normal operation, the control system requires a DC side voltage remains in a stable state, the AC side of the system run as unity power factor, that is, zero reactive power output. Reactive power only with $i_{\text {sq }}$, so that the control objective is to make $i_{\text {sq }}$ fast track its reference value $i_{\text {sqref }}$ without overshoot [9].

Design of active power controller. By the formula (2) shows that

$$
P_{S}=\frac{3}{2} u_{s} i_{s d}
$$

In order to achieve the measured active power quickly and accurately track reference value, active power control use TD and fall function to get the amount of correction of the current $i_{\text {sd }}$ and the formula (4) obtained by calculating an estimated value are summed, as the inner active current reference value $i_{\text {sdref. }}$. The controller consists of TD, NLSEF and formula (4).

Design of TD. In order to achieve reasonable transition process, reducing output overshoot, TD is designed to achieve by the classic inertia. 


$$
P_{\text {ref_TD }}=\frac{k}{T s+1} P_{r e f}
$$

$\mathrm{K}$, $\mathrm{T}$ is the adjustable parameter, in this paper $\mathrm{k}=1, \mathrm{~T}=0.05 . \mathrm{P}_{\text {ref_TD }}$ is the transition process of active power's reference value $P_{\text {ref, }}$, the output of the $P_{\text {ref_tD }}$ act as an input of NLSEF.

Design of NLSEF. According to the error between $P_{\text {ref_ }}$ TD and $P_{\text {meas }}$ (the actual value of the active power), NLSEF controller constructed as follows

$$
i_{\text {sdref }}=k_{1} \text { fal }\left(P_{\text {ref_TD }}-P_{\text {meas }}, \alpha_{1}, \delta_{1}\right)
$$

In this formula, $\mathrm{k}_{1}=1, \mathrm{a}_{1}=0.25 \delta_{1}=0.01$

Design of reactive power controller. By the formula (2) draws the reactive power expression,

$$
q_{s}=\frac{3}{2} u_{s} i_{s q}
$$

When the system is stable and $\mathrm{u}_{\mathrm{sd}}$ almost constant, considering the impact of the system static error, use the fall function to get the amount of correction of the current $i_{\text {sq }}$ and the formula (5) obtained by calculating an estimated value are summed, as the Q-axis current component's reference value.

Design of reception system controller

Due to the size of the load carried by the reception system determines the size of DC side of transmission power, so this paper use DC voltage control of reception system. The whole controller consist of the controller of DC voltage for out ring and the controller of current and reactive power for inner ring [10].

Design of the DC voltage controller. VSC-HVDC system is a typical non-linear, multi-variable, strong coupling system. Ignoring the loss of the converter station itself, so that the AC side of the converter station's power and DC-side power are equal. That

$$
\frac{3}{2}\left(u_{s d}-R i_{s d}\right) i_{s d}+\frac{3}{2}\left(u_{s q}-R i_{s q}\right) i_{s q}=u_{d c} i_{d c}+u_{d c} C \frac{d u_{d c}}{d t}
$$

For the three phase AC power, press the d-axis orientation, $u_{s q}=0$.In order to achieve the converter station run by unit power factor, it need to set $i_{\text {sqref }}=0, i_{\text {sq }}=0$.Ignore the impact of $i_{q}$, The formula (6) can be written as

In this formula

$$
\frac{d u_{d c}}{d t}=-\frac{i_{d c}}{C}+\frac{3\left(u_{s d}-R i_{s d}\right)}{2 C u_{d c}} i_{d c}=f_{1}+b_{1} u
$$

The d-axis current component as

$$
f_{1}=-\frac{i_{d c}}{C}, b_{1}=\frac{3\left(u_{s d}-R i_{s d}\right)}{2 C u_{d c}} u=i_{d c}
$$

$$
i_{\text {sdref }}=k_{3} f a l\left(u_{\text {dcref }}-u_{d c}, \alpha_{3}, \delta_{3}\right)-\frac{f_{1}}{b_{1}}=k_{3} f a l\left(u_{\text {dcref }}-u_{d c}, \alpha_{3}, \delta_{3}\right)+\frac{2 i_{d c} u_{d c}}{3\left(u_{s d}-R i_{d c}\right)}
$$

The $\mathrm{k}_{3} \mathrm{a}_{3} \delta_{3}$ are adjustable parameters.

\section{System's Modeling and analysis of Simulation}

\section{The system's response when the sending system AC voltage changes}

When $\mathrm{t}=0.7 \mathrm{~s}$, the sending system's three-phase AC voltage change from $1 \mathrm{pu}$ to $0.9 \mathrm{pu}$, duration is $0.14 \mathrm{~s}$, and simulation time is $1 \mathrm{~s}$. The simulation curve shown in Figure 2, Figure 3

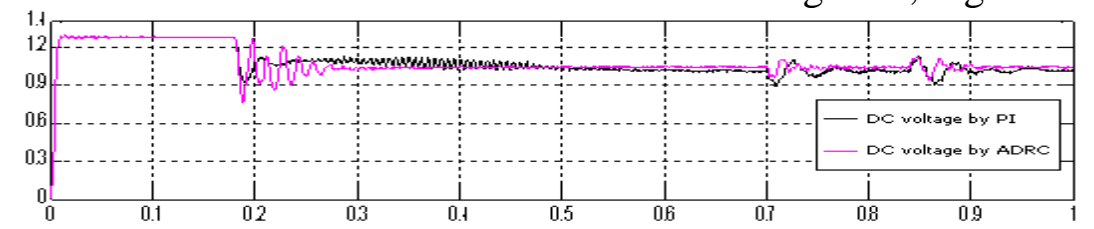

(a)Sending system's DC voltage Udc —meas (Pu) 


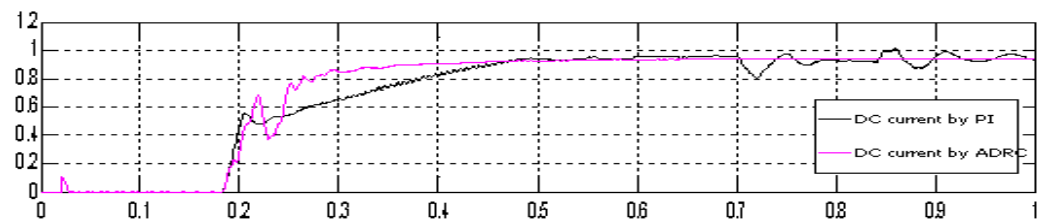

(b)Sending system's DC current Idc —meas (Pu)

Fig.2 Simulation results in inverter when AC voltage changed in inverter side

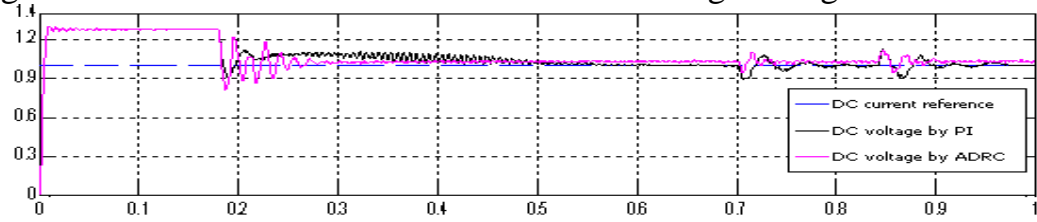

(a) Reception system's DC voltage Udc —meas Uref (Pu)

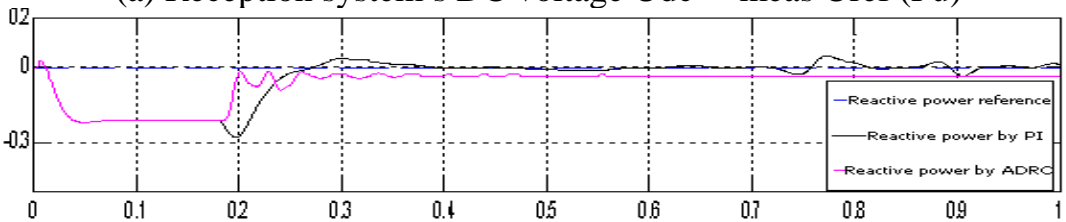

(b) Reception system's reactive power $\mathrm{Q}$-meas $(\mathrm{Pu})$

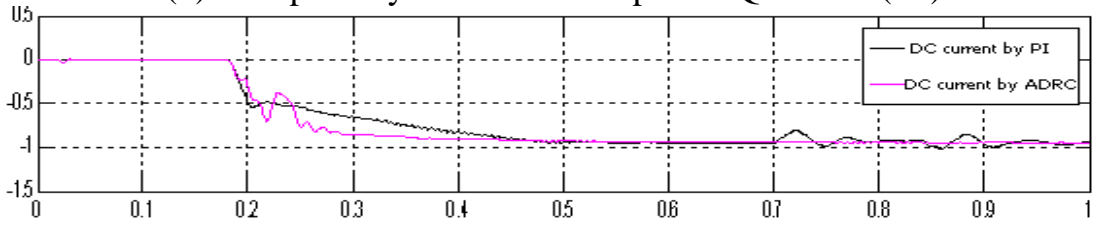

(c) Reception system's DC current Idc-meas (Pu)

Fig.3 Simulation results in rectifier when AC voltage changed in inverter side

As can be seen from FIG. 11 and FIG. 12,when $t=0.7 \mathrm{~s}$, the changes of sending system's AC voltage cause small changes that include the sending system and reception system's DC voltage, DC current, DC-side power and active power.

Sending reactive power have an obvious change. Compared with DC current and reactive power of reception system based on ADRC control, the time to reach steady states is shorter than them based on PI control with less concussion.(FIG. 2(a)(b), FIG. 3(a)(c) and FIG. 3(b))

\section{Conclusions}

In summary, the controller based on ADRC have an easy control method and high steady-state accuracy by using nonlinear function. When the system has a variety of faults, the controller can respond quickly, and adjust the parameters of the system to expected value in the shortest possible time. When the system based on PI starts, its rapidity are slower than ADRC, the stability are worse than ADRC and the immunity are weaker than ADRC. In general, the controller based on ADRC is more suitable for VSC-HVDC system.

\section{Acknowledgements}

The research work was supported by Natural Science Foundation of Shaanxi Provincial under Grant No. 2013JM8016 and Department of Education research project of Shaanxi Provincial under Grant No. 1/JK0986.

\section{References}

[1] ABB. Underground power links by HVDC light. 2008.

[2] ABB Company. It’s time to connect. Technical description of HVDC Light technology. 2005.

[3] Stendius L, Eriksson K.HVDC light: An excellent tool for city centre in feed. Power Gen Conference. 1997. 
[4] Ramadan H S, Signer did Jane H, Petit M.A robust stabilizing nonlinear control design for VSCHVDC system: a comparative study. IEEE International Conference on Industry Technology. 2009.

[5] LI Guangkai, Zhao Chengyong, Geng Ying. Research of noni-near control strategy for VSCHVDC system based on Lyapunov stability theory. Proceedings of Electric Utility Deregulation and Restructuring and Power Technologies. 2008.

[6] Johansson S. G., Asplund G, Jansson E and Ruder all R. Power system stability benefits with VSC DC-transmission systems. CIGRE Session. 2004.

[7] Makoto Hagiwara, Hirofumi Akagi. PWM control and experiment of modular multilevel converters. PESC 2008. 2008.

[8] Guibin Z. Zheng Xu. Steady-State Model for VSC Based HVDC and Its Controller Design [C], Power Engineering Society W intermeeting, 2001,IEEE, Vol 3, 28 Jan. -1 Feb. 2001 Page(s): 1085 1090.

[9] Zhang Guibin, Xu Zheng, Wang Guan. A Linear and Decoupled Control Strategy for VSC Based HVDC System. In: Proceedings of IEEE Power Engineering Society Winter Meeting Vol3. Piscataway (NJ>: IEEE 2001. p. 14-19.

[10] Toshiyuki Hayashi Noboru Seki Masatoshi Sampei etal. Development and Field Test of a Multi-terminal DC Link Using Voltage Sourced Converters and New Control and Protection Schemes .In: Proceedings of CIGRE Session. Paris (France>: CIGRE 2000.14-204. 